had rectal CT. Our study is limited by size and inconsistent documentation of receptive anal sex, however evidence shows this to be a poor predictor of rectal CT infection. We limited our sample to a high prevalence population so our results may not be generalizable to lower prevalence groups.

Disclosure No significant relationships.

\section{P467 FACTORS ASSOCIATED WITH ANORECTAL CHLAMYDIA OR GONORRHOEA TEST POSITIVITY IN WOMEN - A SYSTEMATIC REVIEW AND META-ANALYSIS} 'Jane Hocking. 'University of Melbourne, Melbourne School of Population and Global Health, Carlton, Australia; ${ }^{2}$ The University of Technology Sydney, School of Life Sciences, Ultimo, Australia; ${ }^{3}$ Alfred Health, Melbourne Sexual Health Centre, Carlton, Australia

\subsection{6/sextrans-2019-sti.549}

Background There has been considerable discussion about anorectal Chlamydia trachomatis (CT) in women, but little about anorectal Neisseria gonorrheae (NG). This systematic review and meta-analysis investigates whether anorectal CT in women is associated with detection at other sites (urogenital, oropharyngeal) or anal intercourse and compares this with anorectal NG in the same populations.

Methods Electronic databases EMBASE, MEDLINE and PUBMED were searched for English-language studies published to October 2018 using the search terms: "Chlamydia" OR "Chlamydia trachomatis") AND (("anal" OR "rect"” OR "anorect"”) OR ("extra-genital” OR "multi-site”)). Studies were included if anorectal NG data were available. The primary outcomes, CT and NG positivity, were measured as the proportion of those tested who were test positive. Prevalence ratios (PR) were calculated for the association of anorectal CT or NG with detection at other sites or anal intercourse. Random effects meta-analyses were used to calculate summary estimates; heterogeneity was investigated using meta-regression. Results 25 studies were eligible. Anorectal CT positivity ranged from $0 \%$ to $17.5 \%$ with a summary estimate of $8.2 \%$ (95\% CI: 7.2, 9.2; $\left.\mathrm{I}^{2}=86.4 \%\right)$. Anorectal NG positivity ranged from $0 \%$ to $17.0 \%$ with a summary estimate of $2.2 \%$ (95\% CI: 1.6, 2.8; $\mathrm{I}^{2}=92.6 \%$ ). The association between urogenital and anorectal positivity was stronger for NG than CT $\left(\mathrm{PR}=82.2 \quad\left[95 \% \mathrm{CI}: 50.0,140.9 ; \mathrm{I}^{2}=80.4 \%\right], \mathrm{PR}=29.7 \quad[95 \%\right.$ CI 23.8, 37.1; $\left.\mathrm{I}^{2}=64.6 \%\right]$, respectively). Anal intercourse was associated with anorectal NG $(\mathrm{PR}=4.3$; 95\% CI: 2.18, 8.55; $\left.\mathrm{I}^{2}=0.0 \%\right)$ but not anorectal CT $(\mathrm{PR}=1.0 ; 95 \% \mathrm{CI}: 0.71,1.4$; $\left.\mathrm{I}^{2}=0.0 \%\right)$.

Conclusion Discussion in the literature has focused on anorectal CT in women. This review found that although anorectal CT is more common, anorectal NG is more strongly associated with anal intercourse, urogenital, and oropharyngeal detection. Longitudinal data are required to further understanding of the etiology of anorectal STIs and to inform whether anorectal screening is needed in women.

Disclosure No significant relationships.

\section{P468 THE ASSOCIATION OF SYMPTOMS WITH VIABLE VAGINAL OR RECTAL CHLAMYDIA TRACHOMATIS LOAD: MULTICENTER COHORT STUDY (FEMCURE)}

${ }^{1}$ Kevin Janssen, ${ }^{1}$ Petra Wolffs, ${ }^{2}$ Christian Hoebe, ${ }^{3}$ Titia Heijman, ${ }^{4}$ Hannelore Götz, ${ }^{5}$ Henry De Vries, ${ }^{6}$ Sylvia Bruisten, ${ }^{7}$ Nicole Dukers-Muijrers*. ${ }^{1}$ Maastricht University Medical Centre (MUMC), Department of Medical Microbiology, Care and Public Health Research Institute (CAPHRI), Maastrcicht, Netherlands; ${ }^{2}$ Public Health Service South Limburg, Maastricht University Medical Center (MUMC), Sexual Health, Infectious Diseases and Environmental Health, Medical Microbiology, Care and Public Health Research Institute (CAPHRI), Heerlen, Netherlands; ${ }^{3}$ Public Health Service Amsterdam, Sexual Health, Amsterdam, Netherlands; ${ }^{4}$ Public Health Service Rotterdam Rijnmond, Public Health/Sexual Health, Rotterdam, Netherlands; ${ }^{5}$ Public Health Service Amsterdam, Amsterdam University Medical Center (UMC), National Institute of Public Health and the Environment (RIVM), Infectious Diseases Infection and Immunity Institute (AI and II), Epidemiology and Surveillance Unit, Amsterdam, Netherlands; ${ }^{6}$ Public Health Service Amsterdam, Amsterdam University Medical Center (UMC), Infectious Diseases, Infection and Immunity (AI and II), Amsterdam, Netherlands; ${ }^{7}$ Public Health Service South Limburg, Sexual Health, Infectious Diseases and Environmental Health, Heerlen, Netherlands

\subsection{6/sextrans-2019-sti.550}

Background Symptoms have been associated with Chlamydia trachomatis (CT) infections in culture-based studies, in contrast to studies based on nucleic acid amplification tests (NAAT). This may be because NAAT also detect non-viable bacteria. As culturing techniques are insensitive, we developed a sensitive polymerase chain reaction (V-PCR) technique to measure the viable bacterial load. We here assess the association between symptoms and viable load in 524 women with vaginal or rectal CT.

Methods Prior to treatment at three STI clinics, we included NAAT-CT-positive adult women $(n=411$ vaginal and rectal CT; $n=88$ only vaginal CT; $n=25$ only rectal CT), who were negative for HIV, syphilis and Neisseria gonorrhoeae (Netherlands, 2016-2017; FemCure). We assessed the viable rectal and vaginal $\operatorname{load}\left(\log _{10} \mathrm{CT} / \mathrm{ml}\right)$ using V-PCR. We present the mean viable load (range 0 [non-viable] to 6.5) and tested associations with vaginal symptoms (coital lower abdominal pain, coital blood loss, intermenstrual bleeding, altered discharge, painful or frequent micturition) and rectal symptoms (discharge, pain, blood loss), using multivariable regression techniques adjusting for age and educational level.

Results Of 499 vaginal CT NAAT-positive women, mean viable load was $3.5 \log _{10} \mathrm{CT} / \mathrm{ml}$ (SD: 1.6). Vaginal symptoms were reported by $50.3 \%(n=251)$ of women; women reporting any vaginal symptoms had higher vaginal viable load (mean $3.6 \log _{10} \mathrm{CT} / \mathrm{ml}$ ) than women without symptoms (mean $\left.3.3 \log _{10} \mathrm{CT} / \mathrm{ml}\right) \quad(\mathrm{B}=0.35, \mathrm{p}=0.012) \quad$ (mainly due to 'altered discharge'). Of 436 rectal CT NAAT-positive women mean viable load was $2.2 \log _{10} \mathrm{CT} / \mathrm{ml}$ (SD: 2.0 ); rectal symptoms were reported by $4.8 \%(n=21)$ and not associated with rectal viable load.

Conclusion In an outpatient clinical setting, women diagnosed with vaginal CT have a higher viable load when they have symptoms. Yet, the difference is quite small $\left(0.3 \log _{10} \mathrm{CT} / \mathrm{ml}\right)$ and is therefore unlikely to have a major impact on clinical patient management in women.

Disclosure No significant relationships. 